\title{
Proporción de planes de estudio que abordan la enseñanza de la prevención del confilicto derivado del acto médico en las escuelas y facultades de medicina de México
}

\author{
Moreno Castanedo Hugo*, Figueroa-González Blanca Esthela**, Terrones- Saldívar \\ Ma del Carmen*, Prieto-Macías Jorge*, Rosas-Cabral Alejandro*, Urzúa-Macías Rafael***
}

\section{Resumen}

- La relación médico paciente ha sido considerada el

- acto central de la actividad clínica y el escenario prin-

- cipal de la medicinal. Más allá de los conocimientos

- científicos y desarrollos tecnológicos básicos para la

- práctica médica, e imprescindibles en la formación del

- médico, el diálogo se ha constituido desde la antigüe-

- dad en la base de la entrevista clínica, la guía para

- la exploración del paciente y en el medio terapéutico

- fundamental ${ }^{2}$.Dos factores pudiéramos identificar en el

- deterioro de ésta relación: la vocación y la comunica-

- ción ${ }^{3}$.De esta crisis nace el conflicto derivado del acto

- médico, del cual las facultades y escuelas de medicina

- somos co-responsables. Los instrumentos educativos de

- una buena práctica médica a los estudiantes de medi-

- cina deben otorgarse durante los primeros años de la

licenciatura ${ }^{4}$. El presente trabajo explora el papel de las facultades y escuelas de medicina desde la perspectiva de la educación médica en la búsqueda que contribuya a la humanización de la misma. Se realizaron encuestas a cuarenta y siete facultades y escuelas de medicina, públicas y privadas, asociadas a la AMFEM, con el fin de establecer la enseñanza del conflicto derivado del acto médico.

Los resultados demostraron que el $95 \%$ de las escuelas encuestadas contaban con diversas materias y semestres con los temas de la relación médico-paciente, dilemas bioéticas $u$ otros relacionados con el conflicto derivado del acto médico. En base a los resultados obtenidos se realiza una propuesta de inclusión en los programas de estudio de las Facultades que no cuentan con ello. LUXMÉDICA, AÑO 7 NÚM 21 MAYO-AGOSTO 2012 pp 17-25

Palabras clave: relación médico-paciente, comunicación, conflicto, enseñanza, facultades y escuelas de medicina.

* Profesores investigadores del Centro de Ciencias de la Salud de la Universidad Autónoma de Aguascalientes.

** Estudiante del noveno semestre de la carrera de Medicina de la Universidad Autónoma de Aguascalientes.

*** Comisionado Estatal de Arbitraje Médico para el Estado de Aguascalientes coesamed1@prodigy.netmx

Fecha de recibido: 14 de junio 2012

Fecha de aceptación: 29 de junio 2012

Correspondencia Dr Hugo Moreno Castanedo, Departamento de Medicina, Centro de Ciencias de la Salud, Universidad Autónoma de Aguascalientes. Avenida Universidad 940, CP 2013, Ciudad Universitaria, Aguascalientes, Ags., México. Teléfono 014499108436. Correo electrónico hmoreno@correo.uaa.mx

Este trabajo fue premiado con Mención Honorífica en el III Congreso Internacional de Educación Médica, de la Asociación Mexicana de Federaciones y Escuelas de Medicina (AMFEM) efectuado en mayo 2012 en Puerto Vallarta Jalisco, México. 


\section{Antecedentes}

La relación médico-paciente, sigue siendo, por encima de las continuos progresos en los conocimientos científicos y desarrollos tecnológicos, el sustento de la práctica médica y en consecuencia el escenario fundamental de la formación del médico; sin embargo los cambios históricos han determinado que fuerzas sociales, económicas, políticas, tecnológicas o culturales hayan distorsionado este fundamento esencial, involucrando a los profesionales de la salud en procesos que desvirtúan su misión central ${ }^{2}$.

A lo largo de la historia se ha pensado que el enfermo está incapacitado desde el punto de vista biológico, porque la enfermedad le pone en una situación de sufrimiento e invalidez, de dependencia, y en definitiva, de infantilización.

Históricamente la relación médico paciente entró en su crisis más evidente y profunda con la modernidad 5 , periodo transcurrido entre los siglos XV y XIX, la cual ha sido caracterizada por las transformaciones revolucionarias en todos los órdenes de la vida social dados tres elementos esenciales: la secularización de la vida civil, el poder del desarrollo científico y tecnológico y la transformación política de los estados ${ }^{6}$. El trabajo de algunos médicos, como Frederic Engels y Karl Marx contribuyó a identificar la relación entre trabajo, nivel socioeconómico y enfermedad, así como los efectos de la industrialización sobre las condiciones humanas de la mayoría de la población².

La comunicación entre médico y paciente vigente en occidente se desarrolla a partir de un proceso investigativo, que se consolidó en el siglo XIX bajo apelativo de Método Clínico ${ }^{7}$; éste último basado en el método científico el cual tiene un carácter empírico-experimental, hipotético-deductivo, riguroso y sistemático que conduce al proceder profesional hacia una actitud cognitiva dialéctica y exploratoria valiosa, pero que dentro de los parámetros clási- cos aún vigente se suele centrar en lo orgánico, minorizando fenómenos psíquicos, culturales, espirituales y sociales que son frecuentemente excluidos del análisis clíni$\mathrm{CO}^{2}$. Sigmund Freud (1856 - 1939) supone un cambio muy importante en la relación médico - enfermo al introducir al sujeto en medicina. Considera que en el acto clínico, la relación médico - paciente es fundamental, y la historia clínica un instrumento imprescindible que permite al paciente contar lo que le pasa y relacionarlo con su historia familiar, su trabajo y su vida cotidiana ${ }^{1}$.

Sin embargo era característica de este siglo la concepción del médico considerado un árbitro en la vida del hombre, ejerciendo en régimen de monopolio la alta misión que le fue confiada, regido por sus propios códigos de ética profesional, obligado a respetar el secreto médico y cobrando por su actuación honorarios que el fija libremente ${ }^{8}$.

En nuestros días el ejercicio libre de la profesión es excepcional y el médico ha pasado a estar, en la mayoría de los casos, al servicio de las compañías de seguros o de los grandes sistemas sanitarios colectivizados. Pero el gran progreso de técnicas específicas y la especialización de la medicina en el siglo XX, los mejores recursos de diagnóstico y tratamiento has hecho olvidar nuevamente la subjetividad del enfermo y con ello la relación médico-paciente 9 . Brutti (1991) plantea que el "hecho" tratado por la medicina no tiene relación con la persona, de este modo al paciente no se le reconoce el derecho a la palabra y el médico prefiere obtener la información de aparatos sofisticados que del examen físico y de lo contado por el paciente ${ }^{10}$.

Sin embargo, tendremos sin duda que destacar que la relación médico-paciente no podemos dejar de lado la aplicación de conocimientos y técnicas, esa dimensión técnica también es muy importante pues, sin ella, la relación médico científica con el paciente no se hace bien y el daño puede 
llevar incluso al extremo del deterioro absoluto que se da cuando el paciente fallece y no necesariamente por su propia enfermedad.

El Instituto de Medicina de los Estados Unidos publicó en 1999 una cifra preocupante, estiman, o por lo menos en ese año, que cada año mueren en los Estados Unidos por lo menos 45 mil personas como una situación prevenible, atribuible al médico. Es la consecuencia natural de una relación médico-paciente inaceptable pero posible, cuyo mensaje no solamente se refiere al fenómeno de la comunicación, sino también a la responsabilidad que implica para el médico hacer bien lo que debe hacer, porque de otra manera un error médico puede condenar al paciente a la inexistencia, no solamente de la citada relación sino de su propia persona.

La Comisión Nacional de Arbitraje Médico (CONAMED) es una institución pública en México que ofrece medios alternos para la solución de controversias entre usuarios y prestadores de servicios médicos, también promueve la prestación de servicios de calidad y contribuye en mejorar la seguridad de los pacientes. Desde su creación en Junio de 1996, la CONAMED suma más de 221 mil 239 casos atendidos a un poco más de quince años, asuntos relacionados con las quejas por negligencia médica, inconformidad en los padecimientos, orientaciones y asesorías, por parte de los usuarios del IMSS, ISSSTE y de los servicios de salud pública. De 1996 al 2010, del total de querellas: $61.6 \%$ fue orientación, $21 \%$ asesoría especializada, $10.7 \%$ conciliación o arbitraje, 4\% gestación inmediata y $2.7 \%$ solicitudes de dictámenes médi$\cos ^{11}$.

La Comisión Estatal de Arbitraje Médico de Aguascalientes (COESAMED) reportó 4149 casos atendidos del año 2000 al 2010, según la siguiente gráfica ${ }^{12}$ :

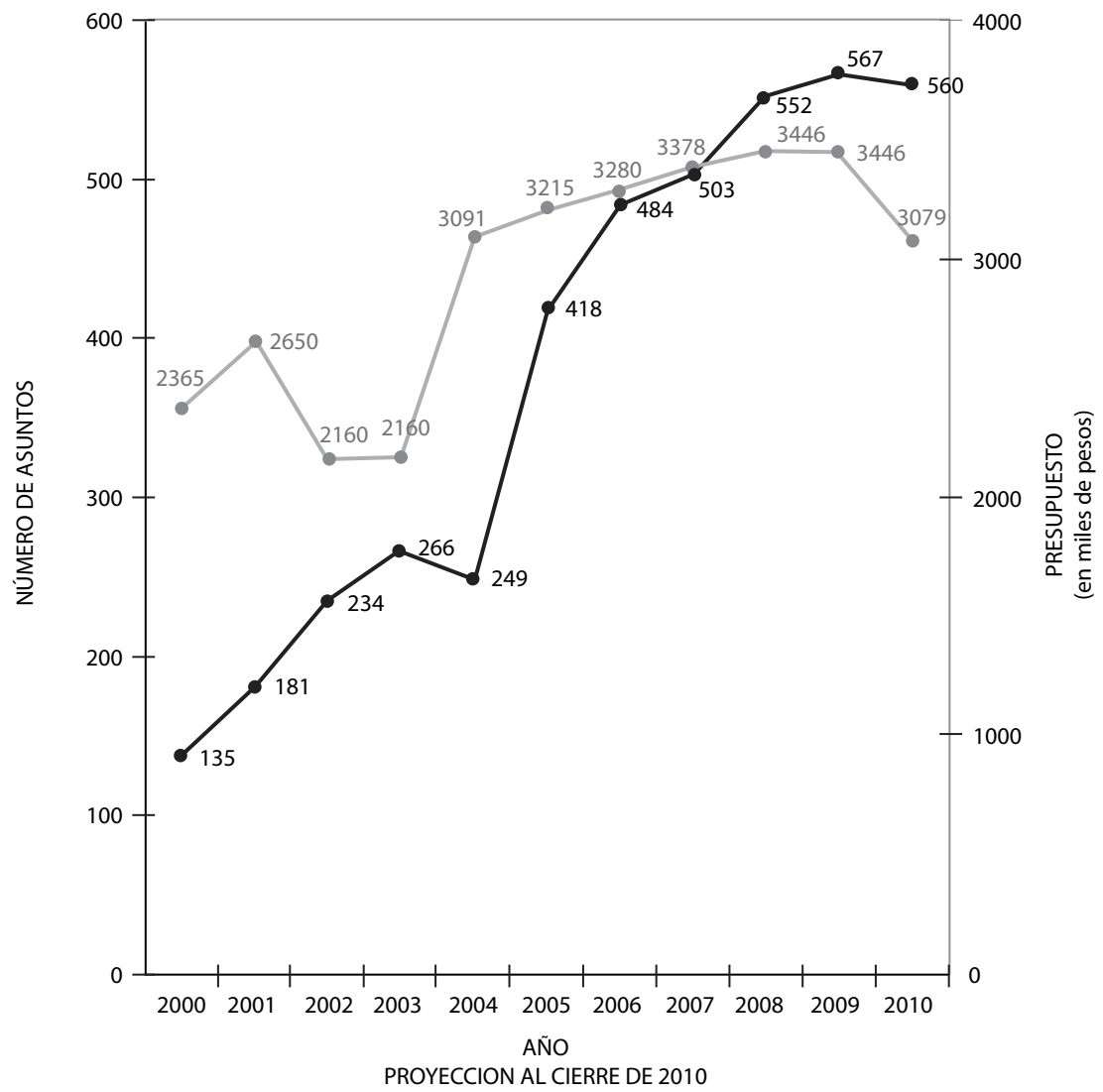


Que como ejemplo, en el año 2011, las diferentes modalidades de asuntos atendidos, se expresan en la siguiente gráfica ${ }^{12}$ :

\section{Total de asuntos atendidos}

$\begin{array}{ll}\text { Orientación/347 } & \text { Asesorías/5 } \\ \text { Gestión inmediata/39 } & \text { Quejas/32 }\end{array}$

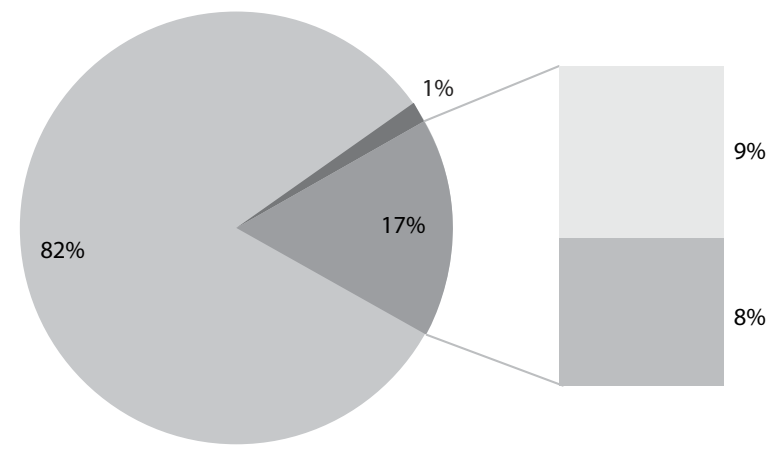

En el mismo periodo, los asuntos atendidos por el tipo de asistencia médica, demostraron que la práctica pública ocupa el $76 \%$, así como en las quejas por especialidad, Cardiología y Cirugía General ocupan los primeros lugares (ver gráficos) ${ }^{12}$.

Asuntos por tipo de asistencia médica

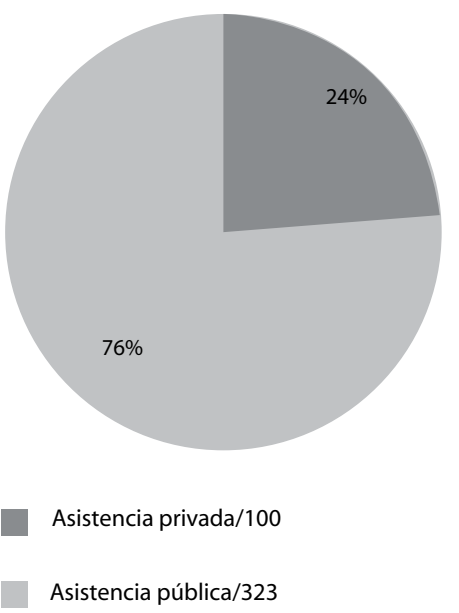

Quejas por especialidad

6

5

4

$\begin{array}{llll}3 & 3 & 3 & 3\end{array}$

2

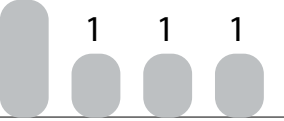

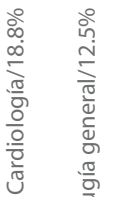

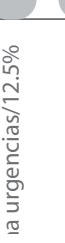

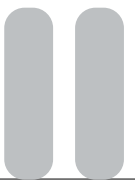

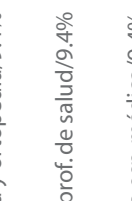


Sin embargo, en el análisis de los errores médicos es imprescindible evaluar tanto al médico como a la institución, ya que la atención a la salud conlleva altas posibilidades de eventos adversos. No obstante, los avances en la medicina, la formación de especialistas e infraestructura tienen tendencia a la mejora de la relación médicopaciente, en un modelo de atención que incluye valores, decisiones e intereses de los enfermos ${ }^{13}$.

El objetivo de este estudio fue explorar la frecuencia de la impartición de materias relacionadas en evitar el conflicto derivado del acto médico, en los diferentes planes de estudio de las Facultades y Escuelas de Medicina de nuestro país.

¿Cómo es hoy la relación médico-paciente? ¿Qué sabemos de la relación médico-paciente en nuestro país? En este contexto tenemos problemas importantes. Encontramos variaciones hasta el $90 \%$ de los criterios diagnósticos y terapéuticos para pacientes en condiciones similares, variaciones que nos hacen pensar que en algunos casos el tratamiento y el diagnóstico seguramente no son muy acertados, pero ¿porqué se da este aparente deterioro en la relación médico-paciente?, pudiéramos identificar factores personales y educacionales, dentro de los primeros el más importante es la vocación y en los educacionales la comunicación.

A los médicos no nos han enseñado a comunicarnos ¿El estudio de la bioquímica, anatomía, fisiología, gineco-obstetricia o de la pediatría per se nos dicen cómo hacerlo? ¿Entendemos lo que significa el fenómeno de comunicación entre los seres humanos? De esta crisis nace el conflicto derivado del acto médico, del cual las facultades y escuelas de medicina somos coresponsables.

\section{| | | | | | | | | | | | | | | | | | | | | | | | | | | | | | | | | | | | | | | | | | | | | | | | | | | | | | | | | | | | | | | | | | | | | | | | | | | | | | | | | | | | | | | | | | | | | | | | | | | | | | | | | | ||}

\section{Metodología}

Se llevó a cabo un estudio descriptivo, transversal y prospectivo. Se aplicó una encuesta a todos los representantes de las diferentes universidades, que se reunieron en el taller "El Docente y la evaluación de las competencias" realizado en Chihuahua, Chih. el 14 de octubre del $2011(n=47)$. La finalidad fue obtener información sobre si se tienen incluidos temas acerca de la relación médico-paciente, sobre dilemas éticos o algún otro relacionado con el conflicto derivado del acto médico en los diferentes planes de estudios de las Facultades y Escuelas de Medicina de nuestro país. Se realizó una base de datos y los resultados se reportaron en frecuencia, porcentajes y promedios.

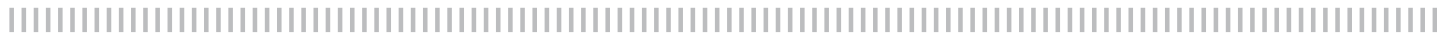

\section{Resultados}

De las 47 facultades y escuelas de medicina encuestadas, el $95 \%$ contaba dentro de sus planes de estudios, temas de la relación médico paciente, dilemas de bioética $\mathrm{u}$ otros relacionados con el conflicto derivado del acto médico (gráfico 1). Dentro de las 47 facultades y escuelas de medicina se analizó, si éstas pertenecían al campo público o privado, encontrándose que la mayoría pertenecen al sector público con un porcentaje de $62 \%$ a comparación del sector privado con $38 \%$ (gráfico 2 ).

La distribución de las materias se muestran en el gráfico 3, donde se observa que la mayor parte de los temas derivados con el conflicto del acto médico se imparten en Bioética Médica con una proporción de $45 \%$, seguida por la materia de Medicina Legal o Aspectos Legislativos con un $30 \%$. 
En cuanto a los semestres en los que se imparten dichas materias, se encontró un mayor porcentaje en la categoría que incluye del $4^{\circ}$ al $7^{\circ}$ semestre con un $37 \%$ del total de nuestro muestreo (gráfico 4).

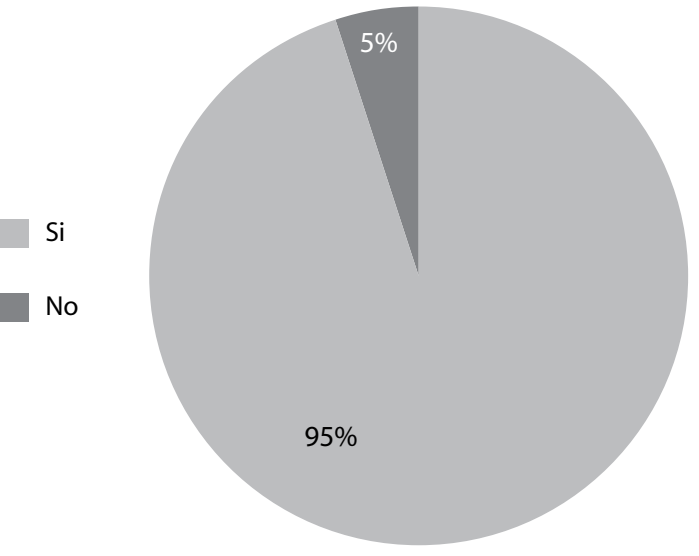

Gráfica 1. Proporción de las instituciones acerca de la inclusión de temas relacionados con la relación médico-paciente o dilemas bioéticos en sus planes de estudio.

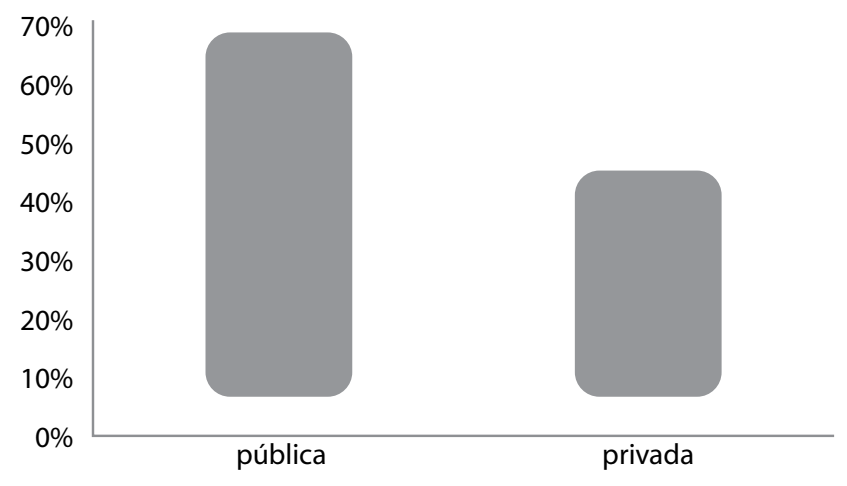

Gráfica 2. Proporción de facultades y escuelas de medicina de acuerdo al sector, sea público o privado, al que pertenecen.

Serie 1

$62 \%$

$38 \%$

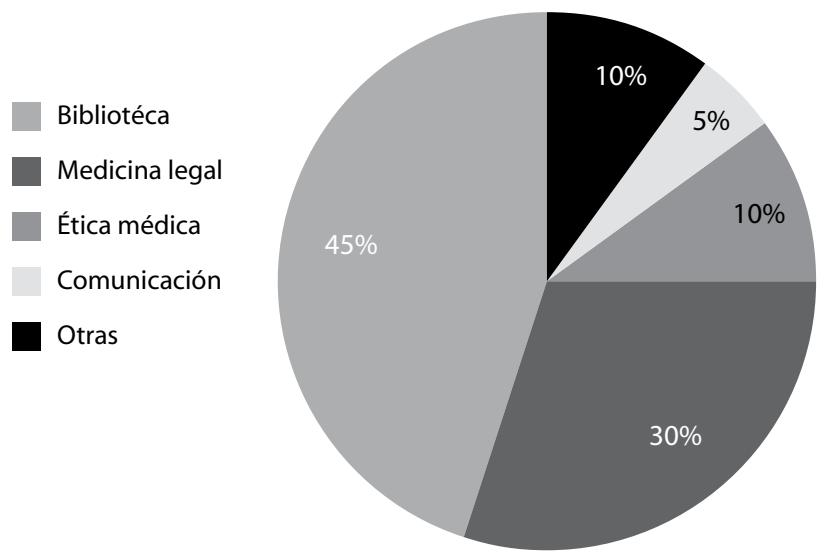

Gráfica 3. Proporción de la distribución de las materias que incluye temas relacionados con el conflicto del acto médico. 


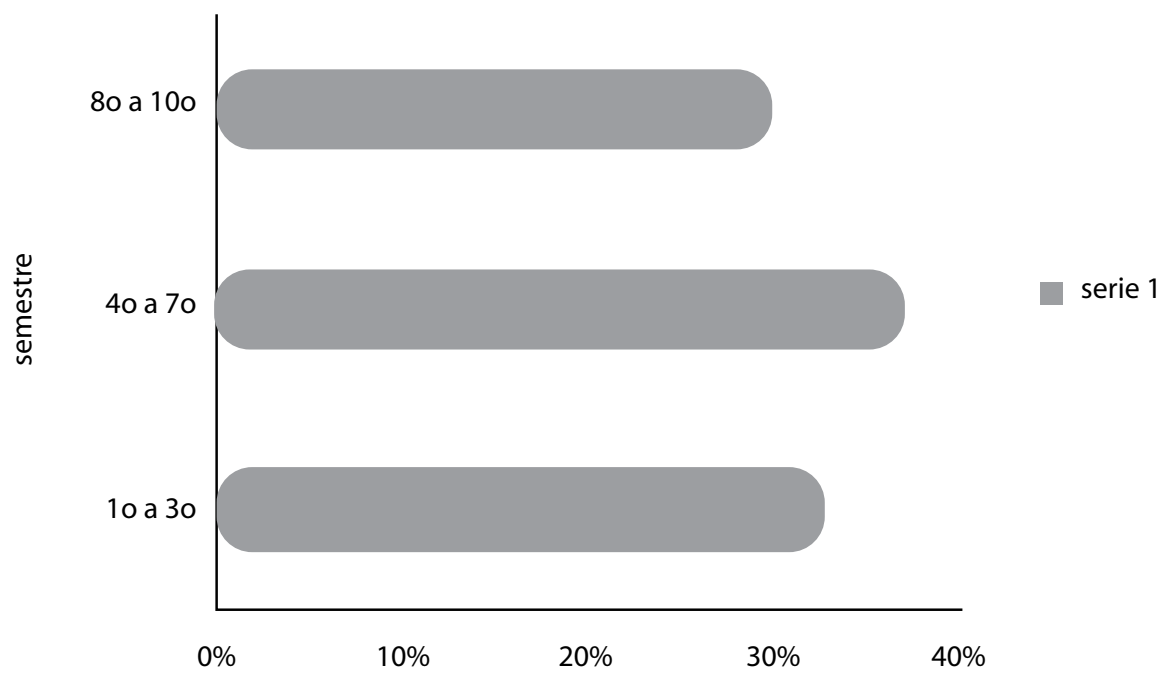

Gráfica 4. Muestra la distribución de los semestres en los que se imparten temas relacionados con el Conflicto derivado del acto médico.

\section{Discusión}

Es evidente que existe un deterioro en la relación médico-paciente dado por factores personales, educacionales, estructurales, tecnológicos y sociales. Dentro de los factores personales el más importante, ya mencionado, es la vocación, y de los educacionales la comunicación, este último factor, muy pobremente enseñado en las facultades y escuelas de medicina del país.

Las instituciones de educación superior, que pertenecen a la AMFEM, donde se imparte la licenciatura de medicina, tienen el reto de ofrecer planes de estudios reconocidos por su calidad. En particular en la Universidad Autónoma de Aguascalientes, existen materias curriculares que pretenden ofrecer al estudiante los valores que deben guiar el acto médico durante su futura práctica médica.

No es muy frecuente señalar la complejidad de la actividad médica y la dificultad de explicar sus peculiaridades a los estudiantes de medicina, sobre todo en los pri- meros años de la licenciatura. No obstante, es importante introducirles a tales complejidades antes de que inicien su periodo clínico a fin de evitar que su primer contacto con los pacientes sea inadecuado debido a que los estudiantes desconozcan lo que los pacientes esperan de sus médicos y la forma de comportarse con aquéllos. En los estudios médicos, la mayoría de los estudiantes han sido formados en enfermedades, no en enfermos. De hecho, la cuestión es considerar la persona enferma antes que exclusivamente la enfermedad.

La comunicación entre médicos y pacientes sigue sin ser la adecuada en muchas ocasiones. Aún hoy no es infrecuente que algunos médicos no contemplen a sus pacientes como una persona, sino como un conjunto de órganos, células y moléculas.

Los resultados de este trabajo nos deben hacer reflexionar sobre el hecho de que, a pesar de que la mayoría de las facultades y escuelas de medicina mexicanas, tienen en su curriculum, materias relacionadas 
con la bioética, las demandas relacionadas con el conflicto médico-paciente se han incrementado, según cifras de la CONAMED11-COESAMED ${ }^{12}$.

De acuerdo con Román y cols. ${ }^{14}$, los valores son completas unidades funcionales cognitivo-afectivas a través de las cuales se produce la regulación de la activación del ser humano; éste mismo autor nos señala que en el sistema de valores del médico general básico se fomenta el humanismo, profesionalidad, responsabilidad, cortesía y sencillez.

Resulta pues imprescindible reorientar las alternativas pedagógicas para ofrecer médicos con una sólida formación disciplinar y humanista.

En general existe la tendencia a ampliar la fundamentación de la bioética en detrimento de la bioética clínica, aunque algunos autores ${ }^{15}$ sugieren, que la enseñanza de la bioética en las facultades de medicina, debe insertarse, como el resto de las asignaturas, en el marco de las competencias.
Según León Correa ${ }^{16}$, algunas acciones educativas para el cambio de actitudes se basa en fundamentar el aprendizaje en casos que descubran no solo métodos de resolución y decisión, sino actitudes en los pacientes y en los equipos de salud, que relacionen lo que enseñamos con lo que suele pasar en sus prácticas, de igual manera proponen usar el método de aprendizaje basado en problemas para el cambio de actitudes.

Por otro lado, como se comentó inicialmente, no se enseña el rol clínico, pedagógico y curativo de la comunicación entre médico y paciente, desarrollada como diálogo y conversación, diferenciándola de procesos como la discusión y otras formas de comunicación menos interactivas y transformadoras.

Con ello, se permitiría descubrir el sentido que tiene la enfermedad, motivando comprensión, acuerdos y aprendizaje recíprocos que hacen de la comunicación médico-paciente un proceso pedagógico dialogante.

\section{Conclusiones}

En conclusión se propone enfatizar y desarrollar explícita y sistemáticamente procesos y prácticas en las facultades y escuelas de medicina y en programas de educación médica continua, que permitan elevar las actitudes y los valores que fomenten el humanismo, así como sustentar la trascendencia formativa que tiene el diálogo en la práctica médica para lograr una mejor prevención del conflicto derivado del acto médico. 


\section{Bibliografía}

1. Laín Entralgo, P. La relación médico-enfermo, historia y teoría. Alianza editorial. Madrid, 1983. p.19

2. Bohórquez, Francisco. El diálogo como mediador de la relación medico - paciente. En: Revista ieRed: Revista Electrónica de la Red de Investigación Educativa [en línea]. Vol.1, No.1 (JulioDiciembre de 2004). Disponible en Internet: http://revista.iered.org. ISSN 1794-8061

3. Ruelas, Enrique. Influencia de las ISES en la Relación Médico-Paciente. Revista CONAMED 2002;7(2):1823 Disponible en http://www.conamed.gob.mx/publicaciones/pdf/rev historico/REV23.pdf

4. Baños, JE. ¿Cuál debe ser el grosor del escudo? La enseñanza de las sutilezas de la relación médico-paciente mediante obras literarias y películas comerciales. Rev. Med. Cine. 3. 2007. 159-165 pps.

5. Laín Entralgo, P. Opus cit, 1983. capítulo III, pp. 218-232

6. Lázaro, J., Gracia, D. La relación médico-enfermo a través de la historia. Anales Sis San Navarra, v. 29, supl. 3. Pamplona, 2006.

7. Bernard, Claude. Introducción al estudio de la medicina experimental. Traducción por José Joaquín. UNAM, México, 1994.

8. Mainetti, José. Bioética médico, III parte: Modernidad, en: Mainetti, J. Ética Médica. Introducción histórica, con documentos de Deontología Médica por Tealdi, Juan Carlos. La Plata, Quirón, 1989 (224 páginas).

9. Focault, M. El nacimiento de la clínica. Una arqueología de la mirada médica. Siglo XXI editores 1989. p. 270

10. Garay, Oscar Ernesto. De la historia clínica tradicional a la historia clínica informatizada. Cuadernos de Bioética (Argentina) No. 4

11. Entrevista al Secretario de Salud José Ángel Córdova Villalobos. www.oportunidadesdenegocios.com.mx/ texto.asp?id_noticia.

12. Reuniones del Consejo de la Comisión Estatal de Arbitraje Médico en Aguascalientes (COESAMED).

13. Entrevista a Dr. José Narro Robles, Rector de la Universidad Nacional Autónoma de México. www.oportunidadesdenegocios.com.mx/texto.asp?id_noticia.

14. Román, Collazo CA. La bioética y la educación en valores en estudiantes de medicina durante la etapa preclínica: un análisis desde el enfoque histórico cultural. Panorama Cuba y salud, 2008; 3 (3): 22-28

15. Couceiro-Vidal, A. Enseñanza de la bioética y planes de estudio basados en competencia. Educmed, 2008; 11(2): 69-76.

16. León Correa, FJ. Enseñar bioética: Como transmitir conocimientos, actitudes y valores. 2008; Acta Bioética 2008; 14 (1): 11-18. 